\title{
Destructions, héritages, CONSTRuCtions. FigurES DE L'EXONOSTALGIE SUR LES FLEUVES DE BELGIQUE FRANCOPHONE.
}

\author{
Laurie Daffe ${ }^{1}$
}

\begin{abstract}
À partir de terrains ethnographiques réalisés en Wallonie et à Bruxelles auprès d'habitants de péniches transformées en habitations, nous envisageons trois questions : Comment les acteurs de terrain se positionnent-ils à l'égard d'un objet de commerce initialement destiné à être détruit et oublié ? Quelles sont leurs attentes? À quels récits, imaginaires et reconfigurations ces attitudes donnent-elles lieu ? En partant de l'analyse des connexions émotionnelles et des attachements entre nouveaux habitants, anciens occupants et bateaux, c'est une lecture de l'exonostalgie - discours et sentiments éprouvés à l'égard d'un passé non vécu personnellement, que nous proposons. Une notion qui nous permettra d'approcher les rapports émiques au patrimoine, à la mémoire, aux transmissions et aux réinventions.
\end{abstract}

Depuis les années 1970 partout en Europe, des centaines de cales sont aménagées en habitations flottantes par des " gens d'à terre », comme on les appelle dans le milieu, dont l'expérience préalable de la vie à bord ou de la batellerie est limitée, voire

1 Laurie Daffe est doctorante FRESH-FNRS au Laboratoire d'Anthropologie Prospective de l'Université catholique de Louvain.

Recherches en communication, $n^{\circ} 46$ - Article publié le 26/06/2018 
inexistante. L'étude de ce phénomène ${ }^{2}$ en Belgique francophone met en évidence une certaine forme d'attirance et de regret exprimés à l'égard d'un univers fluvial inconnu des nouveaux habitants ${ }^{3}$. Une déclinaison de la nostalgie que l'anthropologue David Berliner propose de précéder du préfixe « exo », par opposition à l' " endonostalgie ", nostalgie pour un passé vécu personnellement (Berliner, 2014, p. 375).

Comment l'exonostalgie se manifeste-t-elle chez les acteurs de terrain à l'égard d'un objet de commerce initialement destiné à être détruit et oublié ? Quelles sont les attentes vis-à-vis de cet objet? À quels récits, imaginaires et reconfigurations ces attitudes donnent-elles lieu ? À travers ces questionnements appliqués au phénomène d'habitat fluvial en Belgique francophone, nous montrerons comment la notion d'exonostalgie actualise les notions de patrimoine ainsi que de mémoire.

\section{L'exonostalgie, une madeleine de Proust inversée}

La notion d'exonostalgie repose sur une vision de la nostalgie en tant que sentiment provoqué par le constat d'irréversibilité de la temporalité humaine (Berliner, 2014, p. 375). Cependant, à l'inverse de la nostalgie proustienne, qui incarne une émotion de forte intensité suscitée par la résurgence involontaire d'un passé vécu en personne, l'exonostalgie renvoie aux discours et sentiments éprouvés à l'égard d'un passé non expérimenté

2 Le corpus mobilisé a été constitué pour les besoins d'un mémoire en anthropologie, puis depuis 2014 dans le cadre d'une thèse de doctorat en anthropologie. Il repose sur trois méthodes : d'abord, l'implication de longue durée auprès des acteurs de terrain (« observation participante » et « participation observante »), en tant que secrétaire pour deux associations regroupant des habitants fluviaux - l'une ciblée sur Anderlecht, et l'autre s'étendant à toute la Wallonie - ainsi qu'au cours de séjours à bord de bateaux amarrés sur le Canal Bruxelles-Charleroi, le Canal Blaton-Ath ainsi que sur la Meuse namuroise. Ensuite, 34 entretiens semi-directifs auprès d'habitants fluviaux et de fonctionnaires, ainsi que d'innombrables échanges informels. Enfin, le recensement des zones d'amarrage permanent en Belgique francophone et le relevé architectural de 21 bateaux d'habitation, commentés par les propriétaires et analysés à l'aide des outils de la syntaxe spatiale.

3 La forme masculine est utilisée dans cet article, puisque la très grande majorité des habitants sont des hommes. 
personnellement (Berliner, 2015, p. 21). Une posture qu'Arjun Appadurai qualifie de «nostalgie en pantoufle » (Appadurai, 2005, cité dans Berliner, 2014, p. 375) pour désigner plus largement le regret face à la disparition et à la perte présumées d'un passé, d'un monde, d'une culture... éprouvés par d'autres individus.

Face aux reconfigurations socio-spatiales du $20^{\mathrm{e}}$ siècle que Marc Augé (1992) propose de qualifier de surmodernitét, le concept d'exonostalgie apparait comme particulièrement pertinent. En effet, pour Alfredo González-Ruibal, les processus destructifs sont caractéristiques de cette époque, désormais capable d'en produire à une échelle inédite. Ainsi, ce ne sont plus des villes, mais des continents entiers qui peuvent aujourd'hui être dévastés (Bevan, 2016, p. 18), bien que ces processus soient généralement de nature lente et graduelle, comme en témoigne par exemple la formation de paysages industriels abandonnés (González-Ruibal, 2008, p. 248). Par conséquent, la destruction est constitutive de la réalité matérielle contemporaine au même titre que la production et la consommation. Dans ce contexte, le concept d'exonostalgie en tant que posture émotionnelle et cognitive parait particulièrement opérant à deux égards.

D'une part, d'un point de vue épistémologique ${ }^{5}$, les positionnements théoriques face à l'extinction, la disparition, la perte, le bouleversement... révèlent des exonostalgies profondément ancrées dans l'histoire des disciplines, au " risque d'esthétisation et de romantisation des ruines modernes » (González-Ruibal, 2008, p. 251, notre traduction). D'autre part, cette notion s'avère riche d'un point de vue analytique, pour approcher les rapports émiques vis-à-vis des phénomènes contemporains d'abandon et de destruction, et envisager la manière dont les acteurs concernés s'emparent des notions de patrimoine et de mémoire. Dans les deux cas de figure, c'est donc tout le potentiel créatif du passé qui est mobilisé, plus uniquement sous

4 Terme désignant les reconfigurations socio-spatiales liées à la révolution de la vitesse au $20^{\mathrm{e}}$ siècle, impliquant une intensification des informations, des moyens de communication et de déplacement (González-Ruibal, 2008 : 247).

5 Pour un développement plus complet de cet aspect, voir Berliner, 2014 et Augé \& Berliner, 2015, plus particulièrement en ce qui concerne la discipline anthropologique. 
l'angle des persistances, mais tout à la fois comme réinventions et changements (Berliner, 2014, p. 381).

\section{L'habitat fluvial : reconstruire, ne pas détruire}

Fin des années 1960, la crise charbonnière ainsi que le déclin des activités sidérurgiques, relocalisées auprès des ports maritimes au nord du pays et non plus le long des fleuves wallons (Hanin, 2004), portent un coup dur au secteur du transport par voie d'eau wallon. En outre, l'essor du transport routier est fulgurant, facilité par les programmes nationaux de construction d'autoroutes, tandis que le transport ferroviaire se montre encore vivace. La concurrence est particulièrement rude pour les bateliers propriétaires de bateaux dits de « petits gabarits » ${ }^{6}$, d'une capacité de 200 à 400 tonnes, soit précisément les tonnages convoités par les routiers. Résultat : une quantité importante de cales dévaluées est abandonnée sur des fleuves qui incarnent progressivement les séquelles du processus d'industrialisation (Kinder, 2015, p. 16).

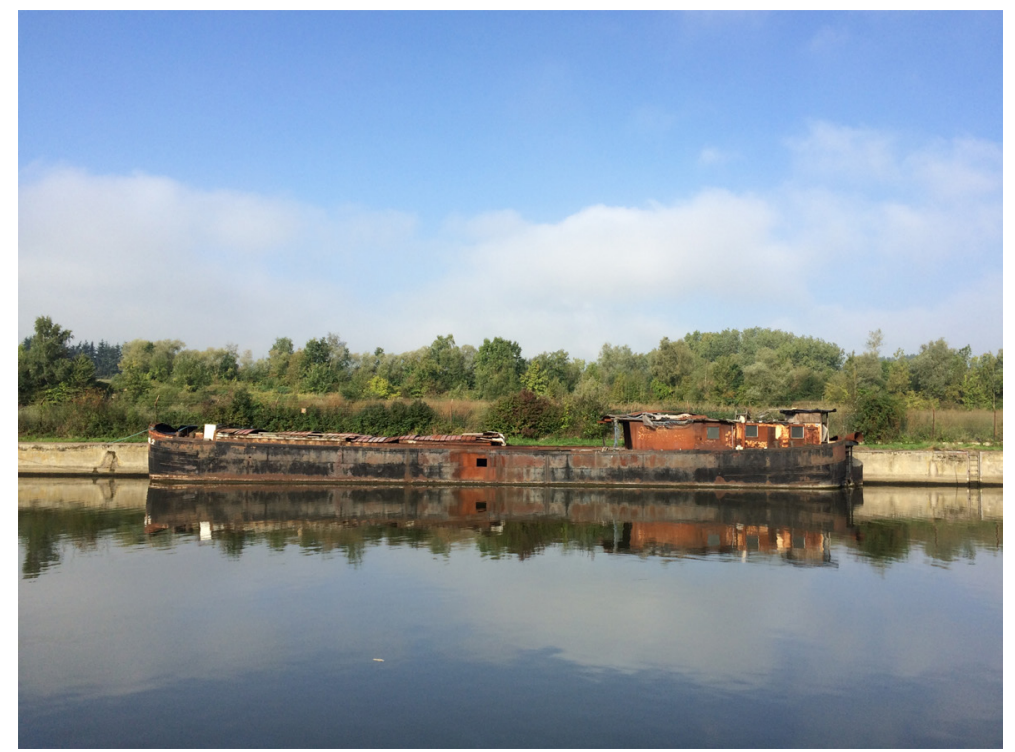

Figure 1. Bateau de 38 mètres partiellement transformé en

6 Bateaux appelés « Spits », « Freycinet », ou plus génériquement « 38 mètres ». 
habitation, amarré à côté du site des anciennes Forges de Clabecq, sur le Canal Bruxelles-Charleroi. Photo de l'auteure.

\subsection{De l'acier-dépassé à l'acier-potentialité}

Suite aux grèves organisées par les syndicats en 1975, le Ministère des Communications et la Fédération des Entreprises Belges instaurent notamment un système de dédommagements financiers pour compenser les mesures « d'assainissement structurel » du secteur, mieux connues sous le nom de « déchirage $»^{7}$, visant à détruire une partie de la flotte de navigation intérieure. Cette mesure connait à l'époque un réel succès : en 1980, on compte 539 demandes de déchirage, soit 93.350 tonnes de plus que la quantité initialement prévue par le gouvernement pour endiguer la surcapacité de cales (Wijnakker, 1982 : 342). En parallèle, une autre option, informelle, vient d'à terre :

On n'a pas trop cherché après une maison. [...] L'alternative c'était d'acheter un bateau vide, un bateau de transport et de le transformer. Et à l'époque, l'Europe incitait beaucoup les bateliers des petits tonnages comme le nôtre à envoyer les bateaux à la casse. À l'époque, on appelait ça le déchirage. Ils payaient des primes donc il y avait beaucoup de bateliers qui se séparaient de leur bateau. Soit par le déchirage, soit en les vendant à des particuliers pour des raisons sentimentales, mais en réalité pour des raisons financières parce que souvent ils en obtenaient plus que ce que le déchirage offrait. Donc voilà. C'est comme ça que nous on a acheté le nôtre. [...] Mais il fallait tout faire dedans, y'avait rien. C'était une cale vide quoi. (C.S., Meuse, 9 mars 2012)

À l'image des nombreuses fermes jugées trop petites et inadaptées aux conditions de l'agriculture moderne, ces cales

7 Il consiste en « la mise à la ferraille intégrale de la coque du bateau » (Règlement (CEE) $n^{\circ} 1101 / 89$, article 5,1 ) ainsi qu'au déchirage des papiers du bateau, d'où le nom de cette mesure. 
vides offrent aux yeux d'une frange de la population un « potentiel de transformation et de rénovation $»$; elles donnent la possibilité à toute une classe moyenne d'après-guerre de choisir un lieu de vie "à leur image » tout en refusant d'adhérer aux logiques de dépendances financières (Daffe, 2016, p. 42).
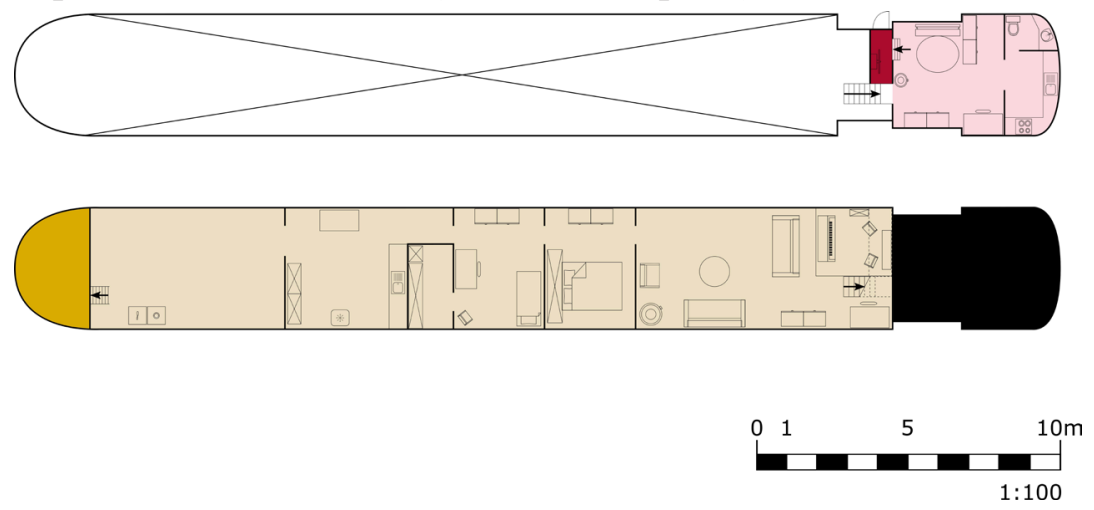

Figure 2. Schéma des transformations apportées à un ancien bateau de commerce converti en habitation. En rose : « roef », ancien logement du marinier et de sa famille (cuisine, salle de bain, salon-salle à manger), ici toujours utilisé par les occupants. En rouge : «marquise », poste de pilotage. En beige : nouvelles pièces de vie (salon, bar, chambres, dressing, atelier, buanderiepièce de rangement). En jaune : «pik avant», ancien logement du matelot, utilisé ici comme atelier. Dessin de l'auteure.

\subsection{La valeur patrimoniale de la destruction}

D'un côté, des cales vides inusitées et disponibles, amarrées le long de terrains industriels inoccupés et, de l'autre, des individus qui cherchent à rompre avec les lieux et modes de vie standardisés (Hanin, 2004 , p. 193). L'habitat fluvial est-il donc le fruit de la rencontre opportune d'une offre et d'une demande ; doit-il son existence à une succession fortuite de hasards ? Ce serait oublier les reconfigurations sociales et culturelles de l'époque et « les 
nouvelles perspectives culturalistes favorables aux patrimoines ${ }^{8}$ hérités » (Hanin, 2004, p. 193).

En outre, dans le contexte socio-politique décrit plus avant et jusqu'à l'heure actuelle, et pour reprendre les termes de Thierry Bonnot, c'est toute la valence du bateau déclassé et envoyé au déchirage qui se reconfigure, à savoir :

[...] l'ensemble de ses liaisons avec les individus, la combinaison de son histoire singulière et collective, l'attraction et la répulsion qu'il a suscité et suscite encore, tout ce qui dans sa biographie a compté pour que cet objet soit conservé et devenu patrimonial. (Bonnot, 2014, p. 188)

Plus encore, tel que le souligne Robert Bevan au sujet des architectures menacées en temps de guerre : leur valeur est rappelée, confirmée et augmente à mesure que des efforts sont déployés pour les détruire (Bevan, 2016, p. 28) ainsi que le laisse entendre - certes à une autre échelle - cette informatrice :

- Enquêtrice : Ce qui t'a plu dans ce bateau-ci, c'est...

- M.K. : ...c'est parce qu'il y avait ce bateau-là qui était là, qu'il fallait sauver, et c'était possible à ce moment-là. [...] Je pense qu'ils [les bateliers] sont quand même contents qu'on mette pas leur péniche à la casse et... C'est marrant comme ça touche le fait de... Ça porte un nom... C'est comment quand on casse une péniche complètement ?!

- Enquêtrice : Le déchirage?

- M.K. : Ha, le déchirage ! C'est un truc qui fait un peu froid à tout le monde. [...] Ça reste du patrimoine industriel, ça fait partie des choses importantes. Je suis attachée à ce côté-là en tous cas. (M.K., Meuse, 15 février 2017)

8 Ceci, en partant du principe selon lequel les catégories développées par les acteurs de terrain sont valides, " y compris quand il y a non seulement hétérogénéité rédhibitoire des membres de ces catégories, mais aussi, éventuellement, une ontologie défaillante lorsque la catégorie renvoie soit à des choses qui ne sont pas ce qu'il est dit qu'elles sont, soit à des choses inexistantes. Bref, l'anthropologue n'est ni gêné ni effrayé par les interprétations «sauvages», «naïves» ou «profanes» du patrimoine » (Candau \& Mazzuchi Ferreira, 2015, p. 27). 
Dans l'histoire de l'habitat fluvial, tout se passe comme si, pour les candidats venant d'à terre, la valence, l'attractivité de l'objet bateau était révélée par et à la mesure des intentions à le faire disparaitre ; le déchirage apparaissant alors aux yeux de certains habitants fluviaux comme « une amnésie forcée à l'égard du groupe en tant que groupe, et sur ses membres individuels » (Bevan, 2016, p. 28, notre traduction). Ainsi, c'est le prisme du déchirage et de son intensité qui soudainement placent le bateau au centre de récits puissants, individuels puis collectifs :

Pour qu'il y ait patrimoine, il faut que les caractéristiques de l'entité (matérielle ou « immatérielle ») candidate à la patrimonialisation puissent être considérées par les membres d'un groupe comme la trace d'une histoire racontable, comme le support d'un récit de soi qui, idéalement, sera reçu, entendu, approprié et partagé par les destinataires de cette histoire et ainsi inséré dans une histoire collective (familiale ou communautaire). (Candau \& Mazzuchi Ferreira, 2015, p. 33)

\subsection{Une mémoire actualisée}

En évitant la casse à une partie de la flotte, c'est également à toute une mémoire menacée d'oubli définitif et contenue dans le bateau, à laquelle les habitants semblent vouloir accéder en même temps que porter secours. D'une certaine manière, Kimberley Kinder $(2015$, p. 1) leur donne raison, à propos d'Amsterdam : pour la chercheuse, les usages informels (habitat fluvial, squats, marchés...) de lieux et bâtiments industriels déclassés ont progressivement contribué à rendre ces infrastructures à nouveau pensables et désirables dans le cadre de projets de redéveloppement des fronts d'eau urbains à large échelle. S'écartant de la définition des «places of abjection », ces endroits ne furent donc jamais complètement condamnés à l'oubli ; leur existence ne fut nullement niée (González-Ruibal, 2008, p. 256). Par conséquent, ils constituent les bases matérielles d'une mémoire reconstituée, ici en relation à un objet ainsi qu'à un groupe socio-professionnel 
perçu comme fragilisé par ceux n'ayant jamais mis un pied à bord d'un bateau avant d'y habiter.

\section{Parmi les décombres, des récits, des pratiques}

Ce n'est donc pas tant à la coque d'acier rouillé qu'il est porté secours, mais aux promesses auxquelles cette image renvoie : de cet objet dont les mesures de destruction confirment l'appartenance à un temps révolu, le nouvel habitant attend qu'il le relie à ce passé, qu'il l'abrite et soit promesse d'avenir (de Botton, 2006, p. 78). Son architecture est « éloquente »; une véritable entrée en dialogue s'opère : "Les bâtiments parlentet de choses aisément discernables. Ils parlent de démocratie ou d'aristocratie, de franchise ou d'arrogance, de bienveillance ou de menace, d'une sympathie pour l'avenir ou d'un regret du passé » (de Botton, 2006, p. 87, italiques originaux). Plus précisément, l'habitant attend d'un bâtiment qu'il lui parle « de ce que nous trouvons important et avons besoin qu'on nous rappelle » (de Botton, 2006, p. 87) ; tandis qu'à son tour le bâtiment parvient à " être aimé » et " à nous convaincre de lui accorder notre confiance " lorsqu'il manifeste sa permanence et démontre qu'il n'est « pas encore mort » en n'offrant pas de surface finie, brillante, univoque (Eno, cité dans Brand, 1994, p. 11).

\subsection{La péniche et ses accroches}

L'attirance pour l'univers du bateau et la nécessité de le « sauver » sont donc moins liées à ses valeurs intrinsèques qu'aux relations qu'il permet d'instaurer : « ce qui importe, c'est l'histoire de l'interaction, les associations que les personnes font avec les objets, et les souvenirs qu'ils évoquent », ce que Donald Norman nomme « connexion émotionnelle » (Norman, 2004, p. 46), mise en mots par cet informateur :

Quand j'étais gamin, j'idéalisais le monde du voyage, donc les forains, les mariniers, et pourtant j'avais même pas de fleuve, y'avait simplement l'Ourthe, donc à part 
des kayaks, je ne vois pas [...] J'ai cherché pour voir d'où me venait cette idée, je pense que j'ai vu le feuilleton L'Homme du Picardie quand j'étais gamin. Je l'ai retrouvé récemment et quand je le revois c'est triste, c'est glauque. Mais moi je zappais cet aspect dramatique, et je voyais plutôt l'aspect du bateau, du voyage et de cette possibilité de se déplacer avec sa maison. [...] (V.L., Meuse, 30 janvier 2012)

Dans cet extrait, V. L. explique ses attentes vis-à-vis d'un bateau : qu'il le transporte, littéralement, mais aussi qu'il le mette en lien avec un milieu ( « le monde du voyage ») approché dans sa jeunesse au travers d'une série télévisée (L'Homme du Picardie, Office de Radiodiffusion Télévision Française, 1968). Dans celleci, le bateau tient ses promesses de voyage, malgré les rudes conditions qui y sont dépeintes, celles du secteur batelier de la fin des années 1960. C'est un événement dans sa vie personnelle (son divorce) qui rappelle à V. L. ce désir d'enfance passé aux oubliettes, et qui le décide à s'investir dans sa réalisation, après une longue période au cours de laquelle il se renseigne et établit des relations, non seulement avec « des gens », mais aussi et surtout avec les péniches elles-mêmes :

Vers 46 ans, je me suis séparé de mon épouse [...] Voilà, et à ce moment-là je me suis dit, tiens ! Il fallait revendre la maison, mais moi je me souvenais quand j'étais gamin, de ce que je voulais faire [...] Avant de m'investir là-dedans, j'ai d'abord été évidemment lié à des choses, rencontré des gens, et puis j'ai vu que je passais beaucoup de temps à m'intéresser à ce sujet-là, que ça redevenait une passion. Et alors je me suis lancé là-dedans. [...] Puis un jour je suis tombé sur la bonne péniche, il m'a fallu plus d'un an, j'en ai vu plus d'une centaine quand même. Maintenant y'en a que je ne suis même pas monté dessus. Rien que [il mime un coup d'œil d'un mouvement de la tête], c'est tout quoi ! (V.L., Meuse, 30 janvier 2012) 
À travers cet extrait révélateur et à la lumière de ce qui vient d'être développé, ce sont en réalité des attachements, entendus tout à la fois comme « ce à quoi nous tenons », « ce qui nous tient », mais aussi « ce qui fait lien » et « le lien que l'on fait » (LeclercOlive, 2015, p. 25), individuellement autant que collectivement, qui sont mis en exergue.

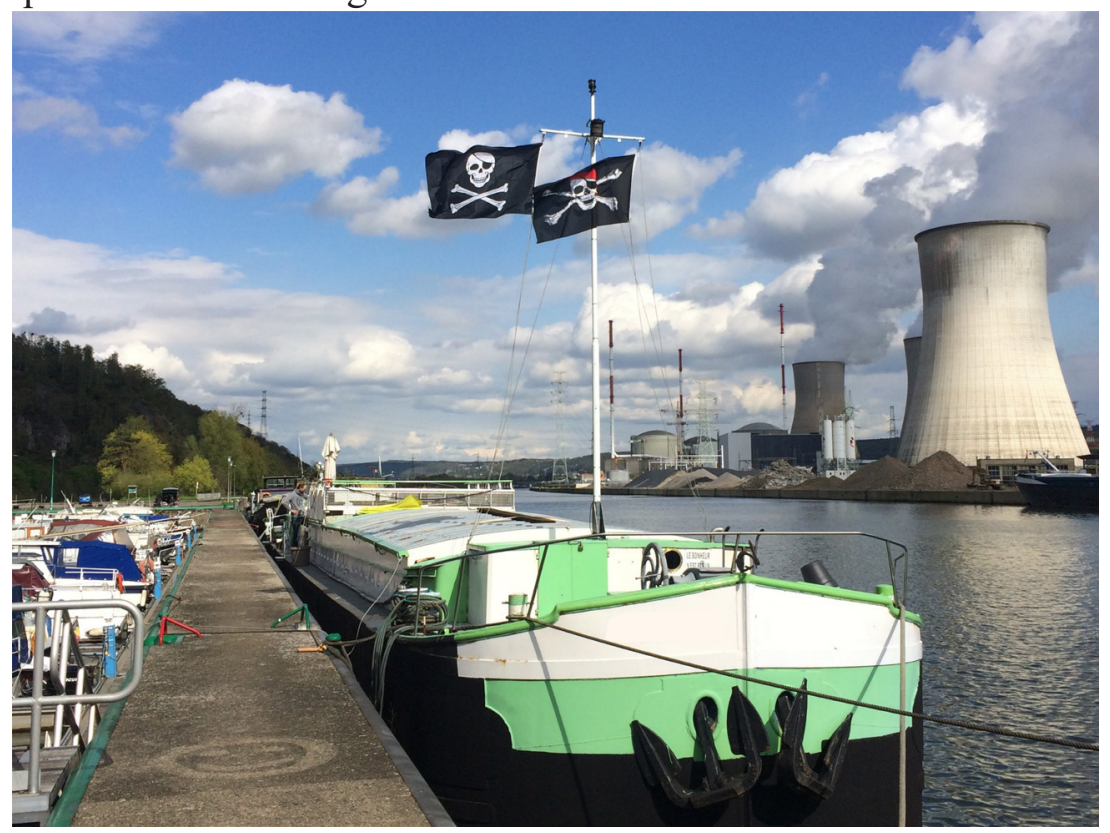

Figure 3. Bateau d'habitation devant la centrale nucléaire de Tihange, sur la Meuse. Photo de l'auteure.

\subsubsection{Attaches biographiques : se (re)trouver}

Dans la coprésence, l'objet bateau implique l'habitant dans sa propre réalisation, et vice-versa. L'investissement personnel du nouvel occupant dans la structure physique du bateau, mais aussi dans la construction de leurs histoires respectives, participe du succès et de la qualité de ces liens. D'une part, le choix d'un bateau particulier n'est jamais laissé au hasard. Rares sont les récits qui n'évoquent pas avec insistance la manière dont l'histoire personnelle du candidat habitant fluvial fait écho à celle du bateau, 
et comment celle des anciens bateliers entre en résonnance avec celle du futur habitant :

Pourquoi est-ce que tout d'un coup, je me suis levé le matin, je me suis dit j'achète une péniche et $\mathrm{j}$ 'ai acheté le [Bernardo] ? Le [Bernardo], il a été construit l'année où je suis né. [Les anciens mariniers] ont un fils qui a le même âge que moi. Il disparait en plongée et puis voilà que moi j'arrive et moi j'achète leur bateau. J'adore la Bretagne, [la marinière] est Bretonne. Enfin, y'a plein de trucs qui font que, c'est assez incroyable quoi. Y'a beaucoup de coïncidences... [rit] (B.O., Meuse, 11 janvier 2012)

D'autre part, si les mises en récit relatent la manière dont les biographies des anciens propriétaires, des nouveaux occupants, et du bateau lui-même se répondent et prennent sens les unes par rapport aux autres, elles mettent également en exergue la façon dont le bateau lui-même a lancé son invitation : " On pouvait voir la coque, il était complètement nu. Je pouvais toucher l'acier, c'est ça qui m'a plu. J'ai dit [satisfaite] : «Ha ! Ça c'est quelque chose de solide ! Avec ça je suis en sécurité !» » (K.A., Meuse, 10 septembre 2017).

Très fréquemment, les nouveaux habitants fluviaux passent les premiers mois (parfois années) de leur vie à bord dans le rouf', ancien logement du marinier et de sa famille. Chambres, cuisine, salle à manger et - pour les mieux lotis - pièce d'eau se partagent l'espace situé en contrebas de la marquise ${ }^{10}$, sur une surface variant de dix à vingt mètres carrés, les deux-tiers restants étant donc entièrement alloués aux cales en attente d'aménagement. Le mobilier intégré et l'étroitesse de ces espaces sur le bateau obligent l'habitant à rapidement s'installer en lui et vice versa. Le rouf devient « l'espace-bulle » de Cousin : un prolongement de l'humain dans l'espace ; «l'espace avec lequel l'homme arrive à s'identifier » et qui est à sa mesure (Cousin, 1980).

$9 \mathrm{Ou}$, suivant les régions et les usages : « roef », « roof » (littéralement : « toit »), « reu », « roufle » ou encore « peak (ou pic) arrière ».

$10 \mathrm{Ou} \ll$ timonerie » : poste de pilotage. 
Un habitant rit en se souvenant que sa stature - deux mètres correspondait exactement à la hauteur sous plafond du rouf, l'obligeant à se déplacer courbé et se cognant régulièrement la tête... Ce qui ne lui laissa donc peu d'autre choix que de rehausser l'ensemble de la structure de son futur logement (B.C., Canal Bruxelles-Charleroi, 19 décembre 2016). Bateau et occupant s'habituent et participent mutuellement à la voluminosité de leurs corps respectifs (Cousin, 1980, p. 23). C'est donc dans cette prise de possession mutuelle que l'habitant apprend en premier lieu ce qu'est un bateau et, plus intimement, qui est son bateau : selon les termes couramment employés, il découvre sa « personnalité », son « âme » voire, dans certains cas, sa « pathologie ».

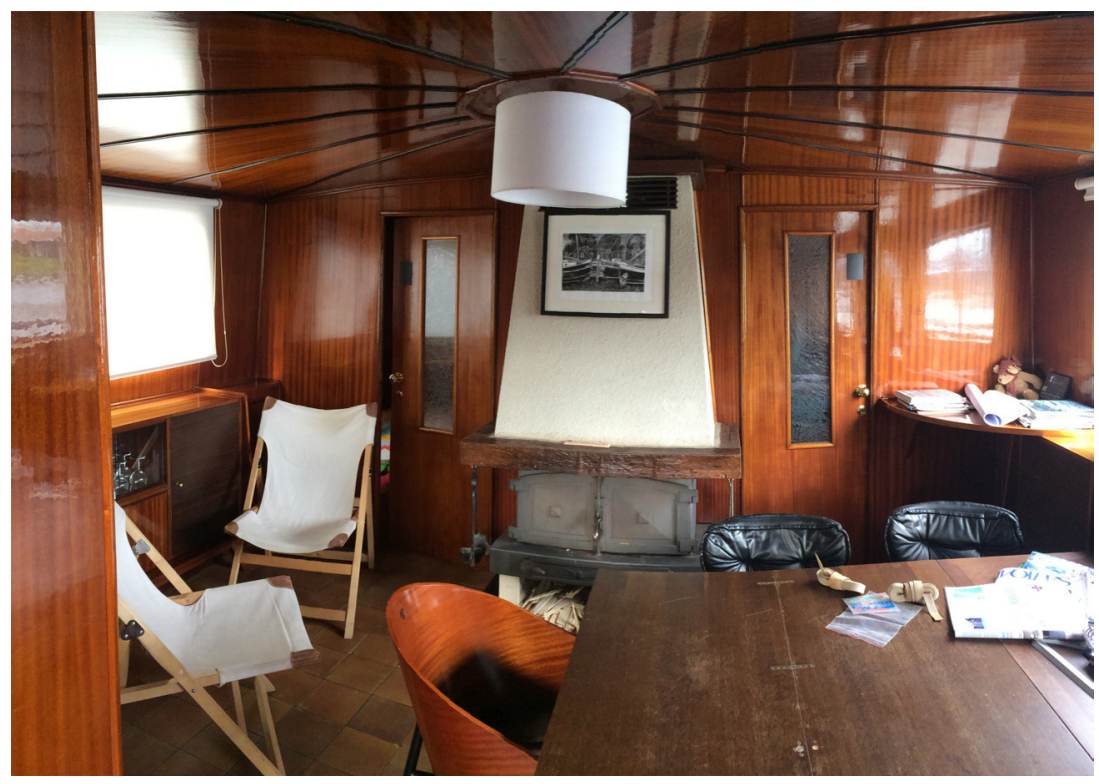

Figure 4. Rouf datant de 1962, dans un ancien bateau de commerce destiné à être transformé en habitation. Photo de l'auteure.

\subsubsection{Attaches gestuelles: s'essayer}

Lors de cette rencontre, 1'habitant fluvial prend aussi connaissance des gestes incorporés à cet outil de travail, inscrits dans la matérialité, la morphologie et l'agencement du bateau, 
littéralement. D'abord, le bateau rappelle sa condition de bateau : l'acier, au contact constant de l'eau, rouille et s'érode ; l'habitant peint et repeint à l'infini, fait changer les cloisons de la coque devenues trop fines. Même transformé de fond en cale, il impose l'emploi d'escaliers pour accéder aux différents niveaux, ainsi que le maintien de bollards ${ }^{11}$ à bord pour l'amarrer à la berge et lutter contre les mouvements du courant ${ }^{12}$. Certains rudiments de la vie à bord se devinent au quotidien, par la manipulation d'éléments dont l'affordance perceptible est mise à l'épreuve des circonstances. Celles-ci tranchent et démontrent si l'intuition était trompeuse... ou non. Quant au vocabulaire, celui d'à terre est utilisé par assimilation dans un premier temps ("volant» pour « macaron », « rouler » pour « naviguer », « sol » pour " plat bord »...) jusqu'à utiliser, à mesure des rencontres et conversations, le jargon fluvial.

Mais la quiétude prend généralement un tournant plus alarmant lorsque l'apprentissage sur le tas connait une issue potentiellement désolante, lors des premières crues par exemple. En de pareilles circonstances, sans connaissance approfondie de la vie à bord, aucun geste ne peut être anticipé et improvisé. Le réflexe est alors généralement de courir chercher un voisin expérimenté ou d'appeler l'ancien propriétaire qui nommera les éléments à manipuler et les étapes à réaliser ${ }^{13}$. Néanmoins, certains habitants moins téméraires négocient lors de la vente quelques heures d'écolage sous la houlette de l'ancien marinier afin de connaitre quelques ficelles du métier.

Malheureusement, les accidents ne sont pas rares. Plusieurs personnes rencontrées ont par exemple perdu un doigt pendant des manœuvres approximativement menées ; d'autres ont provoqué d'importants dégâts, frôlant la collision avec un autre bateau lors

11 Éléments d'amarrage de forme cylindrique, appelés « bittes d'amarrage » dans le langage courant.

12 En effet, sauf obligation (mise en cale sèche, chômage du fleuve...), la grande majorité des bateaux étudiés ne quittent jamais leur emplacement d'amarrage.

13 Les contacts entre parties perdurent en effet généralement de manière épisodique après la passation de propriété, véritables balises dans un mode de vie que ces professeurs, comptables, rentiers, architectes... découvrent en même temps qu'ils y installent leurs meubles. 
d'une panne de moteur ayant entrainé la panique à bord... C'est alors $a$ posteriori, en racontant leurs mésaventures à des fluviaux expérimentés que ces personnes apprendront, à leurs dépens, ce qu'il était indiqué de faire.

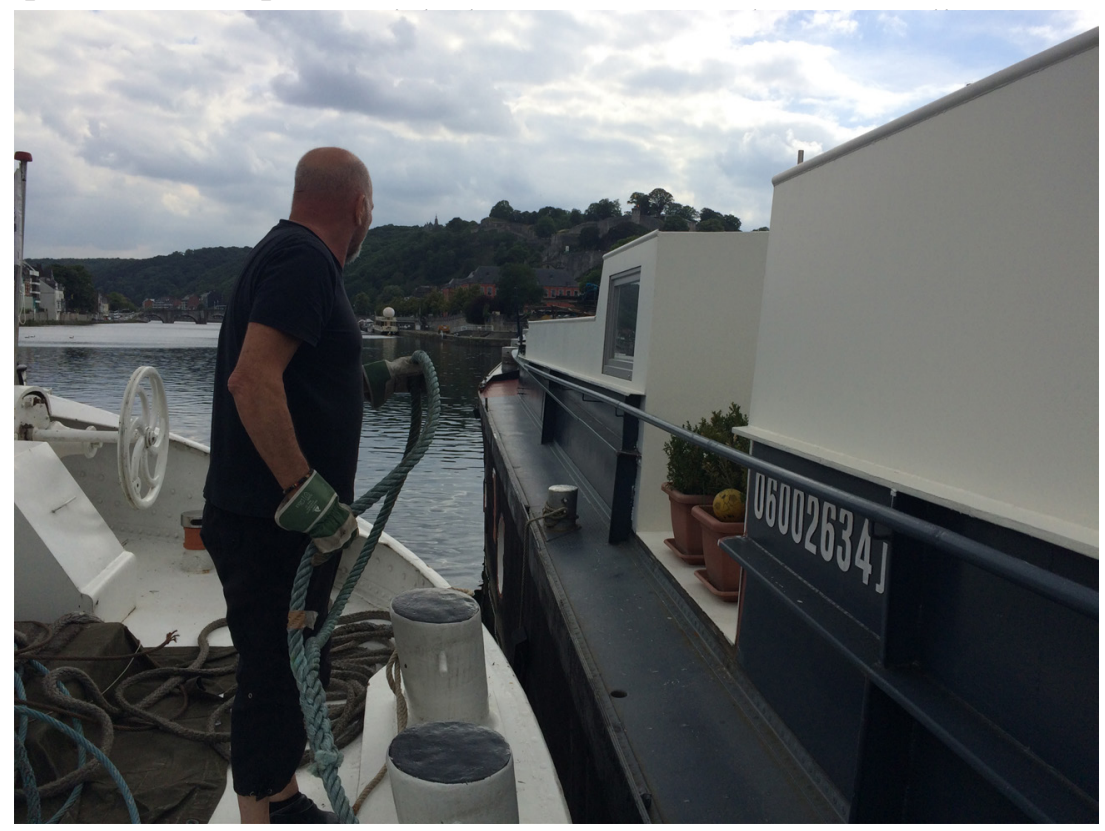

Figure 5. Ancien marinier aidant aux amarres et surveillant les opérations lors d'un amarrage " à couple " (bord-à-bord), l'habitant fluvial étant au macaron (à la barre). Photo de l'auteure.

\subsection{Le mythe de Popeye}

Lors des opérations de requalification des fronts d'eau à Philadelphie, l'accent fut mis sur la valorisation d'un héritage naval préindustriel idéalisé, présenté comme témoignage des vertus nationales, dans lequel « des pans entiers de la mémoire sociale " furent occultés, aseptisés et simplifiés (Giband, 1998, p. 324, cité dans Romain, 2010, p. 63). Un mécanisme d'idéalisation d'un ordre moral passé, similaire à celui observé vis-à-vis de la batellerie artisanale et plus généralement à l'égard des fleuves européens : 
On a vidé le fleuve de son sens historique pour le remplir à nouveau, cette fois d'intentions : la nature du fleuve, c'est ce que nous aurions perdu du passé, et que nous ne retrouverions plus dans le présent trop moderne à notre goût. (Chabenat, 1996, p. 289, cité dans Romain, 2010, p. 289)

Par ailleurs, d'après certains habitants fluviaux, faire l'acquisition d'une péniche de 38 mètres permettrait de renouer avec la solidarité, la lenteur, la liberté et le sens de la communauté dont les bateliers seraient garants, des qualités que les gens d'à terre auraient perdues :

Y'a une solidarité énorme entre tous les gens de la mer et de l'eau. [...] Sur les bateaux, y'a pas de hiérarchie sociale. On rencontre quelqu'un sur un quai et on prend le temps. Enfin, je sais pas si c'est de nouveau l'eau qui fait ça, mais les gens d'à terre, ils arrêtent pas de courir tout le temps, ils sont tout le temps pressés, ils ont jamais le temps. (B.O., Meuse, 11 janvier 2012)

La persistance du terme « péniche » dans le langage commun pour désigner tout bateau de commerce sans distinction, est particulièrement éloquente à ce sujet, en ce qu'il désigne un modèle de chaland aujourd'hui disparu, entièrement construit en bois, sans moteur, et obligatoirement lent, car tracté depuis le chemin de halage généralement par les femmes ou par les animaux. Un mot qui, semble-t-il, « suffit [...] à recréer tout un univers présentant aux yeux des contemporains des garanties d'authenticité suffisantes pour l'ériger en tradition, l'établir comme référence » (Lenclud, 1987, p. 8).

La représentation idéalisée du batelier comme marin nonchalant coiffé d'une casquette et fumant la pipe (une sorte de " mythe de Popeye ») connait encore de beaux jours et tend en outre à occulter les rudes conditions de travail du secteur batelier à l'heure actuelle, qui ne peut se payer le luxe d'être indolent. 


\section{Souffle de vie ou nécessité d'oublier?}

Mais si d'aucuns se félicitent d'avoir évité la perte définitive et irrévocable d'une partie de la flotte, cet engouement ne semble pas unilatéralement partagé par les bateliers. Nous évoquions par exemple en début d'article le succès inattendu du déchirage, qui perdure aujourd'hui dans d'autres conditions. Ne faudrait-il pas y voir la volonté d'oubli de leur outil de travail pour certains mariniers ? C'est ce que laissent entendre certains entretiens :

- Enquêtrice : Et les anciens bateliers sont revenus?

- B.O. : Plus jamais. Je les ai revus, mais ça fait un petit temps que j'ai pas eu de nouvelles. [...] Je leur avais proposé de revenir s'ils en avaient envie ou quand je suis parti pour jeter un coup d'œil, venir passer quelques jours sur le bateau. Ils m'ont dit : non non! Non non! La réponse a été : non. Direct. La page est tournée, c'est... On reste amis, copains, on prend des nouvelles l'un de l'autre, mais le bateau, non : la page est tournée. (B.O., Meuse, 17 décembre 2016)

Joël Candau relève la difficulté à comprendre les attitudes apatrimoniales, telles que la destruction volontaire d'outils de travail par les paysans eux-mêmes, comme nécessaires au travail de mémoire : "Alors qu'[...] une dimension essentielle de la mémoire est l'oubli, il semble que dans le domaine du patrimoine, l'angoisse de la perte conduise à tout garder et à ne rien vouloir oublier » (Candau, 2005, p. 120). Or, l'auteur l'affirme : la destruction de ces engins peut constituer une étape essentielle pour rejeter « hors de leur mémoire [des] objets évoquant pour eux un métier pénible, fait de souffrances, de dureté et d'efforts, permettant tout juste de «gagner sa vie»» (Candau, 2005, p. 122).

\section{Conclusion}

C'est à partir de la notion d' « exonostalgie » que nous avons proposé une lecture des récits et pratiques liés à la destruction de bateaux de commerce de petit gabarit. En Belgique francophone, 
l'habitat fluvial nait dans un contexte où ces péniches, déclarées désuètes pour le marché, se révèlent être, aux yeux de personnes extérieures à la batellerie, les témoins à sauvegarder d'une époque industrielle révolue, autant qu'une opportunité d'habitat. En évitant la casse à une partie de la flotte, c'est également à des valeurs et à toute une mémoire menacée d'oubli définitif et contenue dans le bateau, à laquelle les habitants semblent vouloir accéder en même temps que porter secours. Nous avons alors montré comment s'établissaient les liens et relations entre nouveaux habitants, anciens propriétaires, et le bateau lui-même, relevant la manière dont à la fois les biographies respectives s'imbriquaient, certaines pratiques étaient partiellement transmises et persistaient et, enfin, comment tout un passé était actualisé par ces habitants qui attendaient d'y être transportés... Pour terminer, il convient de souligner que, bien que la flotte des 38 mètres ait drastiquement diminué en Belgique au cours des 60 dernières années, le petit gabarit de commerce n'est pas mort. Il connait même un regain d'intérêt à l'heure actuelle pour certains types de frets, comme l'acheminement de pieds d'éoliennes par exemple, ou les transports de matériaux intra-urbains. Qui plus est, comme nous venons de le décrire dans cet article, il connaît désormais une existence à part entière, en parallèle de sa vie commerciale, sous forme de logement.

Quoi qu'il en soit, qu'il soit acheté auprès de bateliers en fin de carrière, ou sur le marché conventionnel de l'immobilier, le 38 mètres habité se situe à l'interface « d'intérêts particuliers et d'enjeux collectifs »(Bonnot, 2014, p. 10). Il donne prise à l'actualisation d'un passé anonyme, mais aussi aux réinterprétations multiples quant à sa morphologie et quant à sa destination. De cette manière, l'acier déclassé devient acierpotentialité ; de mains en mains, le bateau continue à circuler.

\section{Références}

Berliner, D. (2014). On exonostalgia. Anthropological Theory, 14(4), pp. 373-386.

Berliner, D. (2015). Are Anthropologists Nostalgists? Dans O. Angé \& D. Berliner (Éd.), Anthropology and Nostalgia (pp. 17-34). New York : Berghahn Books. 
Bevan, R. (2016 [2006]). The destruction of memory. Architecture at war. Londres : Reaktion Books.

Bonnot, T. (2014). L'attachement aux choses. Paris : CNRS Éditions.

Brand, S. (1994). How buildings learn. What happens after they're built. New-York : Penguin Books.

Candau, J. (2005). Anthropologie de la mémoire. Paris : Armand Colin.

Candau, J., \& Mazzuchi Ferreira, M. L. (2015). Mémoire et patrimoine. Des récits et des affordances du patrimoine. Educar em revista, 58, pp. 21-36. doi: 10.1590/01044060.43469 .

Cousin, J. (1980). L'espace vivant. Introduction à l'espace architectural premier. Paris : Éditions du Moniteur.

Daffe, L. (2016). Trente-huit mètres sur cinq. Genèse du logement fluvial à Bruxelles et en Wallonie. Uzance, 5, pp. 35-44.

de Botton, A. (2006). L'architecture du bonheur. Paris : Mercure de France.

González-Ruibal, A. (2008). Time to Destroy. An Archaeology of Supermodernity. Current Anthropology, 49(2), pp. 247-279.

Hanin, Y. (2004). Mutations spatiales et recompositions territoriales. Les processus territoriaux dans le cas de Court-Saint-Etienne. Louvain-la-Neuve : Presses Universitaires de Louvain.

Kinder, K. (2015). The Politics of Urban Water. Changing Waterscapes in Amsterdam. Georgia : The University of Georgia Press.

Lenclud, G. (1987). La tradition n'est plus ce qu'elle était. Sur les notions de traditions et de société traditionnelle en ethnologie. Terrain, 9, pp. 2-13.

Leclerc-Olive, M. (2015). Au-delà des épistémologies sédentaires. Parcours anthropologiques, 10(1), pp. 24-45.

Norman, D. A. (2004). Emotional Design. Why we love (or hate) everyday things. New York : Basic Books.

Romain, F. (2010). La construction contemporaine des paysages fluviaux urbains (le cas de deux villes nord méditerranéennes : Perpignan et Montpellier. Thèse doctorale, AgroParisTech. Disponible à : https://tel.archives-ouvertes.fr/pastel-00565209/ document

Wijnaker, R. (1982). Transports Fluviaux de Marchandises. Dans Rapports introductifs et synthèse du Symposium international sur la théorie et la pratique dans l'économie des transports, pp. 340-363.

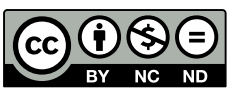

Publié sous la licence Creative Commons

«Attribution - Pas d'Utilisation Commerciale - Pas de Modification 4.0 International» (CC BY-NC-ND) 